\title{
Association of Single Nucleotide Polymorphism in Pancreatic Lipase Gene with Growth-Related Traits in Japanese Black Beef Cattle
}

\author{
${ }^{1}$ Hideki Tanomura, ${ }^{2}$ Youji Muramatsu, ${ }^{1}$ Takuji Yamamoto, ${ }^{3}$ Takeshi Ohta, \\ ${ }^{4}$ Hiroyuki Kose and ${ }^{1}$ Takahisa Yamada \\ ${ }^{1}$ Laboratory of Animal Genetics, Graduate School of Science and Technology, \\ Niigata University, Nishi-ku, 950-2181 Niigata, Japan \\ ${ }^{2}$ Department of Nutritional Sciences for Well-Being, Faculty of Health Sciences for Welfare, \\ Kansai University of Welfare Sciences, Kashiwara, 582-0026 Osaka, Japan \\ ${ }^{3}$ Central Pharmaceutical Research Institute, Japan Tobacco Inc., Takatsuki, 569-1125 Osaka, Japan \\ ${ }^{4}$ Department of Life Science, Division of Natural Sciences, International Christian University, \\ Mitaka, 181-8585 Tokyo, Japan
}

\begin{abstract}
Growth performance as well as marbling is the main breeding objectives in Japanese Black cattle, the major beef breed in Japan. Researchers have recently reported that a Single Nucleotide Polymorphism (SNP) referred to as rs 41648172 in intron 6 region of the Pancreatic Lipase (PNLIP) gene was associated with marbling in Japanese Black beef cattle with the $\mathrm{T}$ allele being associated with a high level of marbling. The PNLIP is known to be involved in the hydrolysis of dietary triglycerides to fatty acids which is essential for the intestinal absorption of long-chain triglyceride fatty acids. Thus, the PNLIP was considered as a functional candidate for the gene responsible for growth performance. In this study, researchers analyzed the effect of the PNLIP rs 41648172 SNP genotypes on the growth-related traits in Japanese Black beef cattle. The SNP was associated with carcass weight, rib eye area, rib thickness and daily gain in the experiment using 367 paternal half-sib progeny steers from a sire homozygous for C allele at the rs 41648172 in Japanese Black beef cattle. The SNP was also associated with carcass weight, rib eye area and daily gain in the experiment using 587 paternal half-sib progeny steers from a sire homozygous for $\mathrm{T}$ allele at the rs 41648172 in Japanese Black beef cattle. These findings suggest possible effect of the rs 41648172 on the growth-related traits in Japanese Black beef cattle. The PNLIP SNP polymorphism may be useful for effective marker-assisted selection to increase the beef productivity in Japanese Black beef cattle.
\end{abstract}

Key words: Association, beef cattle, growth-related trait, Japanese Black breed, single nucleotide polymorphism, PNLIP

\section{INTRODUCTION}

Growth performance has an important effect on the economics of beef production (JMGA, 1998). Thus, it is greatly interesting to obtain better knowledge on the molecular architecture of growth characteristics and to generate new opportunities for more effective markerassisted selection.

Researchers have recently reported that a Single Nucleotide Polymorphism (SNP) referred to as rs 41648172 in the intron 6 region of the Pancreatic Lipase (PNLIP) gene was associated with marbling in Japanese Black beef cattle with the $\mathrm{T}$ allele being associated with a high level of marbling (Tanomura et al., 2011). The PNLIP SNP seems to be a candidate marker for marker-assisted selection of marbling in Japanese Black beef cattle (Tanomura et al., 2011). The PNLIP is known to be involved in the hydrolysis of dietary triglycerides to fatty acids which is essential for the intestinal absorption of long-chain triglyceride fatty acids (Lowe, 2002). Thus, the $P N L I P$ gene was regarded as a functional candidate for the gene responsible for growth performance. Researchers herein analyzed association of the PNLIP SNP, rs41648172 with the growth-related traits in Japanese Black beef cattle.

\section{MATERIALS AND METHODS}

Samples and data: Researchers performed two experiments for the association study. In experiment 1, 367

Corresponding Author: Takahisa Yamada, Laboratory of Animal Genetics, Graduate School of Science and Technology, Niigata University, Nishi-ku, 950-2181 Niigata, Japan 
paternal half-sib Japanese Black progeny steers produced from a sire homozygous for C allele at the rs 41648172 with dams considered to represent a random sample of the female population were used. In Experiment 2,587 paternal half-sib Japanese Black progeny steers produced from a sires homozygous for $\mathrm{T}$ allele at the rs 41648172 with dams considered to be a random mating population were used. These progeny steers were fattened and shipped to a carcass market in the Oita prefecture. Adipose tissues of the progeny steers were collected for SNP genotyping. DNA samples were prepared from the materials according to standard protocols.

The growth-related carcass traits, carcass weight, rib eye area and rib thickness were measured on carcasses dissected at the 6 th and 7 th rib section according to the Japanese meat grading system by certified graders from the Japan Meat Grading Association (Tokyo, Japan) (JMGA, 1998). In addition, researchers used the growth-related trait of daily gain. The predicted breeding values of the sires and the progeny steers for carcass weight, rib eye area, rib thickness and daily gain were obtained from the Oita recording system for beef cattle previously reported by Sasaki et al. (2006). In the recording system, the breeding values were predicted from the growth-related trait records of Japanese Black steers and heifers, fattened in the Oita prefecture. The fattened animals were shipped to various carcass markets from 1988-2003 where they were slaughtered and their carcasses evaluated. The data were edited to connect across subclasses such that each market year subclass had 50 or more animals and each farm had 10 or more animals. The final number of animals was 48,045 and there were 89 market year subclasses, 332 farms and 228 sires.

Data were analyzed by the REML method using the MTDFREML programs (Boldman et al., 1995) and genetic and environmental variances were estimated. The BLUP option in the programs using the estimated variance components was chosen to predict the breeding values of animals with a single trait model. Sex, market year and farm were considered fixed effects. Fattening period and slaughter age were also considered as up to quadratic covariates. The fattening period denotes the period from the start of fattening to shipping to market for each animal. These fixed effects were all significant $(p<0.001)$. Random effects included the additive genetic effect of the individuals that is the animal model was adopted to predict the breeding values.

This study conformed to the guidelines for animal experimentation of the Graduate School of Science and Technology, Niigata University (Niigata, Japan).

SNP genotyping: We genotyped 1 SNP, rs41648172 in dbSNP which is located in the intron 6 region of the
PNLIP. The SNP was genotyped using PCR-Restriction Fragment Length Polymorphism (RFLP) Method. PCR primers used for PCR-RFLP were 5'-CAGTGCTATCTCCCGGAGTC-3' and 5'-GAAATCTAGGTGGCCCACAA3'. PCR amplifications were performed using $25 \mathrm{ng}$ of the prepared DNA as template in a final volume of $25 \mu \mathrm{L}$ containing $0.5 \mu \mathrm{M}$ of each primer, $0.2 \mathrm{mM}$ of each dNTP, $0.625 \mathrm{U}$ of Ex Taq polymerase (Takara, Shiga, Japan) and $1 \mathrm{X}$ Ex Taq buffer (Takara). The PCR conditions were as follows: $94^{\circ} \mathrm{C}$ for $3 \mathrm{~min}, 35$ cycles of $94^{\circ} \mathrm{C}$ for $50 \mathrm{sec}, 66^{\circ} \mathrm{C}$ for $50 \mathrm{sec}$ and $72^{\circ} \mathrm{C}$ for $50 \mathrm{sec}$, followed by a further $5 \mathrm{~min}$ extension at $72^{\circ} \mathrm{C}$. An aliquot of PCR-amplified product was digested at $37^{\circ} \mathrm{C}$ for $1 \mathrm{~h}$ with restriction enzyme $\mathrm{HpyCH} 4 \mathrm{IV}$ and electrophoresed on a 3.0\% agarose gel. Agarose gels were stained with ethidium bromide and photographed under an ultraviolet light.

Statistical analyses: The populations of the two experiments were separately analyzed. The effect of genotypes at the rs41648172 SNP on the predicted breeding values for carcass weight, rib eye area, rib thickness and daily gain was analyzed with the model that included the SNP genotype as the fixed effect in Experiment 1 and 2. Statistical analysis was performed by the GLM procedures (Experiment 1 and 2) of the SAS program (SAS Institute Inc., Cary, NC).

\section{RESULTS AND DISCUSSION}

The populations of the two experiments were separately analyzed for association of the PNLIP SNP with carcass weight, rib eye area, rib thickness and daily gain.

Experiment 1: Genotyping 367 progeny steers from a sire homozygous for the $\mathrm{C}$ allele at the rs41648172 SNP revealed 163 animals homozygous for $\mathrm{C}$ allele and 204 animals heterozygous for $\mathrm{C}$ allele and $\mathrm{T}$ allele. The steers in this experiment could be grouped only according to the alleles that they received from their dams which are considered to be a random sample of a general population in Japanese Black beef cattle. Therefore, this experiment likely allowed a linkage disequilibrium estimate of the effect of the SNP. Statistically significant differences among the genotypes of the SNP were detected for carcass weight $(p=0.0069)$, for rib eye area $(p=0.0017)$, for rib thickness $(p=0.0108)$ and for daily gain $(p=0.0305)$ (Table 1). The carcass weight, rib eye area, rib thickness and daily gain were significantly higher in the CT heterozygotes than in the CC homozygotes (Table 1).

Experiment 2: To verify the association of the rs 41648172 with carcass weight, rib eye area, rib thickness and daily 
Table 1: Effect of the SNP genotypes on growth-related traits in Experiment 1

\begin{tabular}{lccccr}
\hline & & Breeding value $^{1}$ & & & \\
& & & & \\
Genotype & No. of animals & Carcass weight $(\mathrm{kg})$ & Rib eye area $\left(\mathrm{cm}^{2}\right)$ & Rib thickness $(\mathrm{mm})$ & Daily gain $(\mathrm{g})$ \\
\hline CC & 163 & $-4.80 \pm 1.30^{\mathrm{a}}$ & $2.80 \pm 0.21^{\mathrm{a}}$ & $0.28 \pm 0.19^{\mathrm{a}}$ & $3.82 \pm 2.88^{\mathrm{a}}$ \\
CT & 204 & $-0.06 \pm 1.16^{\mathrm{b}}$ & $3.69 \pm 0.19^{\mathrm{b}}$ & $0.95 \pm 0.17^{\mathrm{b}}$ & $12.22 \pm 2.58^{\mathrm{b}}$ \\
\hline
\end{tabular}

Table 2: Effect of the SNP genotypes on growth-related traits in Experiment 2

\begin{tabular}{lccccc} 
& & Breeding value $^{1}$ & & \\
Genotype & No. of animals & Carcass weight $(\mathrm{kg})$ & Rib eye area $\left(\mathrm{cm}^{2}\right)$ & Rib thickness (mm) & Daily gain $(\mathrm{g})$ \\
CT & 283 & $3.78 \pm 1.04^{\mathrm{a}}$ & $3.86 \pm 0.17^{\mathrm{a}}$ & $0.57 \pm 0.15$ & $25.27 \pm 2.42^{\mathrm{a}}$ \\
TT & 304 & $8.88 \pm 1.03^{\mathrm{b}}$ & $4.56 \pm 0.16^{b}$ & $0.97 \pm 0.15$ & $34.52 \pm 2.40^{b}$ \\
\hline
\end{tabular}

${ }^{1}$ The breeding values are given as least squares means \pm SE. ${ }^{a, b}$ Means at different genotypes without a common letter in their superscripts significantly differ $(\mathrm{p}<0.05)$

gain, we carried out Experiment 2. Genotyping 587 progeny steers from a sire homozygous for the $\mathrm{T}$ allele at the rs 41648172 SNP revealed 304 animals heterozygous for $\mathrm{C}$ allele and $\mathrm{T}$ allele and 283 animals homozygous for $\mathrm{T}$ allele. The steers in this experiment could be also grouped only according to the alleles that they received from their dams which are considered to be a random sample of a general population in Japanese Black beef cattle. Therefore this experiment as well as Experiment 1 likely allowed a linkage disequilibrium estimate of the effect of the SNP. The SNP genotype effect reached significance for carcass weight $(p=0.0005)$ for rib eye area $(p=0.0025)$ and for daily gain $(p=0.0061)$ and the SNP genotype effect reached marginal significance for rib thickness $(p=0.0582)($ Table 2$)$. The carcass weight, rib eye area and daily gain were significantly higher in the TT homozygotes than in the CT heterozygotes (Table 2) and the rib thickness exhibited a tendency to be higher in the TT homozygotes than in the CT heterozygotes (Table 2).

On the basis of two experiments using the 367 progeny steers from a sire homozygous for the $\mathrm{C}$ allele at the rs $41648172 \mathrm{SNP}$ and the 587 progeny steers from a sire homozygous for the $\mathrm{T}$ allele at the rs $41648172 \mathrm{SNP}$, researchers showed that the rs 41648172 SNP is associated with carcass weight, rib eye area, rib thickness and daily gain in Japanese Black beef cattle with the $\mathrm{T}$ allele at the rs 41648172 SNP resulting in high levels of carcass weight, rib eye area, rib thickness and daily gain. Especially in Experiment 1 and 2 because the dams can be considered to represent a random sample of the Japanese Black population, the association is likely to be true. We used the predicted breeding values as phenotypic values and then sire contributions to the predicted breeding values were the same for all offspring. Thus, it is likely to be desirable to use larger numbers of dams in the analyses using paternal half-sib progeny steers such as Experiment 1 and 2 . We should note that the experiments in this study were the case.
Based on the association of the rs 41648172 with the carcass weight, rib eye area, rib thickness and daily gain, together with PNLIP function (Lowe, 2002), we can hypothesize that the SNP in the intron 6 region might have an impact on PNLIP function and/or expression and also the carcass weight, rib eye area, rib thickness and daily gain.

However, the SNP is not located within any of as yet identified canonical sequences involved in gene function and/or transcription. Thus, a more likely event is that the PNLIP SNP is in linkage disequilibrium with an unidentified and truly relevant mutation, rather than functional and a causal mutation for the carcass weight, rib eye area, rib thickness and daily gain.

The association with carcass weight, rib eye area, rib thickness and daily gain obtained in this study suggested possible effect of the rs 41648172 on the growth-related traits in Japanese Black beef cattle. The information on the PNLIP SNP may be applied to effective marker-assisted selection to increase the beef productivity in Japanese Black beef cattle.

Setoguchi et al. (2009) and Yamada et al. (2011), respectively, have recently reported that SNPs in the chromosome condensation protein $G$ gene and in the titin gene were associated with growth-related trait in Japanese Black breed. Thus, the present study seems to be the third report to show polymorphisms associated with growthrelated trait using Japanese Black breed.

\section{CONCLUSION}

In this study, researchers show that the rs 41648172 SNP in PNLIP is associated with carcass weight, rib eye area, rib thickness and daily gain in Japanese Black beef cattle. This study will provide an useful information for the establishment of effective marker-assisted selection to increase beef productivity in Japanese Black beef cattle. 


\section{ACKNOWLEDGEMENTS}

This research was supported by a Grant in Aid for Scientific Research (B) (No.: 14360166) from the Ministry of Education, Culture, Sports, Science and Technology of Japan and by the research funds of Japanese Livestock Technology Association.

\section{REFERENCES}

Boldman, K.G., L.A. Kriese, L.D. Van Vleck, C.P. Van Tassell and S.D. Kachman, 1995. A Manual for Use of MTDFREML, A Set of Programs to Obtain Estimates of Variances and Covariances. US Department of Agriculture, Agricultural Research Service, Washington, D.C., USA.

JMGA, 1998. New Beef Carcass Grading Standards. Japan Meat Grading Association, Tokyo, Japan.

Lowe, M.E., 2002. The triglyceride lipases of the pancreas. J. Lipid Res., 43: 2007-2016.
Sasaki, Y., T. Miyake, C. Gaillard, T. Oguni and M. Matsumoto et al., 2006. Comparison of genetic gains per year for carcass traits among breeding programs in the Japanese Brown and the Japanese Black cattle. J. Anim. Sci., 84: 317-323.

Setoguchi, K., M. Furuta, T. Hirano, T. Nagao, T. Watanabe, Y. Sugimoto and A. Takasuga, 2009. Cross-breed comparisons identified a critical $591-\mathrm{kb}$ region for bovine carcass weight QTL (CW-2) on chromosome 6 and the Ile-442-Met substitution in NCAPG as a positional candidate. BMC Genet., 10: 43-43.

Tanomura, H., T. Yamamoto, Y. Muramatsu, T. Ohta, H. Kose and T. Yamada, 2011. The Pancreatic lipase gene is associated with marbling in Japanese black beef cattle. J. Anim. Vet. Adv., 10: 1719-1723.

Yamada, T., S. Sasaki, S. Sukegawa, Y. Takahagi and M. Morita et al., 2011. Possible association of single nucleotide polymorphism in titin gene with growthrelated trait in Japanese black beef cattle. J. Anim. Vet. Adv., 10: 2603-2606. 